\title{
Reliability evaluation for colliery machine based on fuzzy interval model
}

The mechanical reliability design method is a common method, and it is the most direct and effective method to carry out the mechanical reliability design at present. The reliability optimization design is an effective optimization design method which is developed in combination with the reliability design theory on the basis of the conventional optimization design. Taking the antifriction bearing as an example, this paper systematically expounds all kinds of mechanical reliability design methods. Through comprehensive analysis of various factors that affect the reliability of colliery machine, the index system of colliery machine reliability evaluation is established. Because of the complexity and diversity of the use of colliery machine in the evaluation system, and the fuzziness of human thinking, it is difficult to give the deterministic evaluation information in numerical form, so this paper also analyzes the design method of colliery mechanical reliability, and puts forward an interval fuzzy evaluation method for colliery mechanical reliability evaluation to avoid overload operation of colliery machine and ensure safety production and safety of workers. The simulation results show that the inherent law of reliability is effectively characterized by this method, and it provides a evidence for security produce and scientific decision-making of colliery mechine.

Keywords: Reliability evaluation, colliery machine, antifriction bearing, Weibull distribution model, fuzzy interval

\subsection{Introduction}

$\mathrm{T}$ The level of equipment in colliery machine is uneven. The equipment is very backward due to the problems of capital and enterprise concept in some old mines and the expansion mines (Chen and Lee, 2014). The modern mechanized comprehensive mining equipment are adopted in some mines, but the equipment lack normal maintenance for long overloading operation, which leads to the lower equipment reliability. Therefore, it is of great significance to establish the reliability evaluation system of colliery machine and evaluate the reliability of colliery machine. Meanwhile it

Ms. Li Yang and Mr. Chuang Chen School of Equipment Engineering, Shenyang Ligong University, Shenyang, China. is of great significance for the colliery production enterprises, the safety supervision department and the production enterprises of the colliery machine.

With the improvement of reliability in basic theory and standard system, the modern colliery machine reliability engineering technology has entered a mature stage, and has made some achievements (Karthikeyan and Vasuki, 2016). The colliery machine reliability standard system is becoming more and more perfect (Pahlavani, 2017). The colliery machine reliability engineering has basically followed the American standard system. Standardized technology can make RMS (reliability, maintainability and supportability) advanced technology and successful engineering experience widely used. With the development of RMS engineering and management (Messaoud, Bouzid and Ellouze, 2016), the relevant standards have played an important role in improving the efficiency of the colliery machine and reducing the life cycle cost.

A complete colliery machine reliability parameter system has been established, which should reflect the equipment for readiness, mission success, maintenance manpower, support cost requirements of equipment reliability, and reliability parameter of the equipment described by a single variable for a plurality of reliability parameters, the reliability parameters of a complete system of expression of the reliability of products, and the general reliability parameter of the equipment based on MTBF. (Butt and Akram, 2016). The colliery machine reliability parameters are generally divided into basic reliability, task reliability and mission profile. According to the fault judgment, it should be divided into three categories, basic function, maintainance function and add function. The reliability of colliery machine design is a commonly used method. It is the most direct and effective way to carry out colliery mechanical reliability design at present. Because the material strength distribution and load distribution of specific data are scarce, the reliability index is designed to meet the requirements of proper extent of parts.

\subsection{Design method of colliery machine reliability}

The method of colliery machine reliability design can be adopted because of the difference of the product and the difference of its composition. 
Mechanical products generally belong to the series system. To improve the reliability of the whole machine, the strict selection and control of the parts should be started (Ghondaghsaz, Fatemeh, Rasekh and Abdolrahman, 2012). According to the standard selection and the control of the purchased parts the full use of the results of fault analysis, the mature experience or the analysis test is used to verify the scheme. Under the condition of meeting predetermined functions, mechanical design should be simple and the number of spare parts should be reduced as much as possible. Simpler and more reliable are a basic principle of reliability design. It is the most effective way to reduce faults and improve reliability. But it is not possible to make other parts perform super normal functions or work under high stress conditions because of the reduction of parts. Otherwise, the simplified design will not achieve the purpose of improving the reliability.

The reduction design is a design method to make the use stress of the parts less than the rated stress. The reduction design can be achieved by reducing the stress of the parts or improving the strength of the parts. Engineering experience has proved that most mechanical parts work under the rated load stress condition, the failure rate is low, and the reliability is high. In order to find the best value of the drop, a lot of experimental research is needed to do. When the mechanical components of the load stress and bearing stress of the concrete component strength is uncertain distributed in a certain range, the design can be used to improve the average strength and reduce the average stress and reduce the stress changes. the intensity change and other methods can improve reliability. For important parts involving safety, the limit design method can also be adopted in order to ensure that it does not fail in the worst limit state.

Redundancy design is the design of repeating structure, spare parts and other parts to complete the specified function, so that the whole machine or system will still not lose the specified function when the local failure occurs. The redundancy technique may become a kind of design method of a horn, for example, the mechanical system of double pump or twin engine configuration, but it should be noted that the redundancy design will make the weight and cost increased. The redundancy design improves the mission reliability of the mechanical system, but the basic reliability is correspondingly reduced, so the redundancy design should be taken carefully.

Environmental design considering all kinds of environmental impacts that the product may encounter during the whole life cycle, for example, the impact of assembly, transportation, vibration, storage, temperature, humidity, mould, and so on. Therefore, it is necessary to choose the design scheme carefully and take the necessary protection measures to reduce or eliminate the harmful effects of the environment. Specifically, it can be considered from three aspects: understanding the environment, controlling the environment and adapting to the environment. The understanding of the environment not only pay attention to the working environment and maintenance environment of the products, but also to understand the environment of the installation, storage and transportation of the products. In the design and test process, two environmental conditions of single environment and combined environment must be considered. We should not only care about the natural environment of products, but also consider the environment induced by the use process. The control environment means that when conditions permit, a good working environment condition should be created for the designed parts in a small range, or artificially change unfavorable environmental factors for product reliability. Adapting to the environment means that measures should be taken in designing, material selection, surface treatment and coating protection when human control is not available for all environmental conditions, so as to improve the ability of mechanical parts and withstand environmental problems.

The purpose of man-machine engineering design is to reduce the errors in the use of the people, and to improve the reliability of the mechanical products by giving full play to the characteristics of the human and machine. Of course, human error is closely related to human misoperation, except for human reasons, such as control platform, control and control environment. Therefore, man-machine engineering design is to ensure the reliability of the system. Due to misoperation prevention design the control system is reliable on the instrument and machinery satisfactory precision, and it is suitable for people's habits to facilitate the identification operation, less error prone, more concerned with safety.

Robust design is the most representative method for colliery machine, and it is an optimization design by a full consideration in the design process of virtual interferential effects of its reliability. Probability design method is based on the theory of stress and intensity interference. The stress and intensity are treated as random variables which obey certain distribution according to the theory of stress and intensity interference. In order to get the best results the trade-off design refers to a comprehensive tradeoff among reliability, maintainability, safety, functional weight, volume, cost and so on.

With the development of computer technology, simulation method is becoming more and more perfect. It can not only be used for reliability quantitative design of mechanical parts, but also for system level reliability quantitative design. Of course, mechanical design method of mechanical reliability design cannot leave the traditional and some other optimization methods, such as mechanical computer aided design and finite element analysis. 


\subsection{Reliability of colliery machine design}

Reliability design of colliery machine is based on the failure mode influence and hazard analysis, and applies the successful design experience to make the designed product achieve a reliable purpose. The reliability of colliery machine can be divided into structural reliability and mechanism reliability. The structural reliability mainly considers the mechanical strength as well as fatigue and the effect of load on the abrasion and fracture caused by failure. The failure mechanism reliability is the main consideration in the course of action for the problems caused by kinematics.

3.1. The Relationship BETWEen the LIFE OF THE ROLLING BEARING AND THE MECHANICAL RELIABILITY

A mathematical model of Weibull distribution has two parameters. After a large number of experiments, it is proved that the life distribution of the rolling bearing is approximately obeying the two parameter of Weibull distribution, and the failure distribution function of the Weibull distribution is for the Weibull distribution.

$$
F(t)=1-\exp \left[-(t / \eta)^{\beta}\right]
$$

The density function of Weibull distribution is

$$
f(t)=F(t)=(\beta / \eta)(t / \eta)^{\beta-1} \exp \left[-(t / \eta)^{\beta}\right]
$$

The reliability function is

$$
R(t)=\exp \left[-(t / \eta)^{\beta}\right]
$$

The inefficiency function is

$$
r(t)=(\beta / \eta)(t / \eta)^{\beta-1}
$$

The scope of application of Weibull distribution model.

When the shape parameter is $\beta<1$, the reliability of products increase with time and improve the products, suitable for early fault occurs; when $\beta>1$, reliability increase with time and decrease applicable due to aging or wear products and failure; when $\beta=1$, the reliability of the time change is fixed with the same, for a constant, applicable to random failure.

The life of the rolling bearing is subject to the two parameter Weibull distribution, so the reliability is

$$
R(L)=e^{-\left(\frac{L}{b}\right)^{\mathrm{K}}}
$$

The $\mathrm{L}$ is the bearing life, the $\mathrm{b}$ is the dimension parameter of the bearing life distribution, and the $K$ is the shape parameter of the bearing life distribution

$$
R(L)=b\left(\ln \frac{1}{R}\right)^{\frac{1}{K}}
$$

The parameters $b$ and $K$ of Weibull distribution can be obtained, and the relationship between reliability and life span can be obtained. The Weibull parameter is related to reliability. After statistical processing, the parameter data is listed in Table 1.

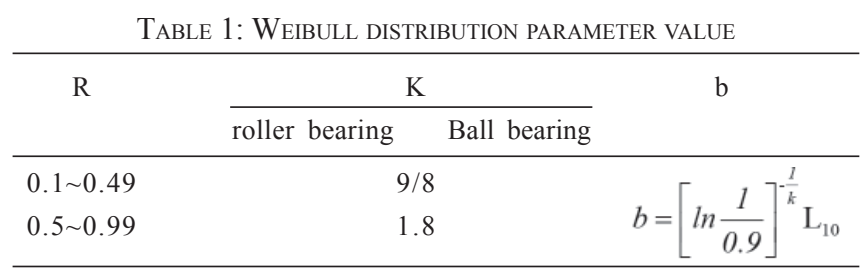

When

$b=\left[\ln \frac{1}{0.9}\right]^{-\frac{1}{k}} \mathrm{~L}_{10}$

Then

$$
\begin{aligned}
& L(R)=\left(\frac{\ln R}{\ln 0.9}\right)^{\frac{1}{K}} L_{10} \\
& \alpha_{1}=\left(\frac{\ln R}{\ln 0.9}\right)^{\frac{1}{K}} \\
& L(R)=\alpha_{1} L_{10}
\end{aligned}
$$

The type $\alpha_{1}$ is the reliability coefficient, and the $L_{10}$ is the basic rated life of the bearing.

\subsection{RATED DYNAMIC LOAD AND MECHANICAL RELIABILITY OF ROLLING BEARING}

The relationship between the rated dynamic load and the basic rated life

$$
L_{10}=\left(\frac{C}{P}\right)^{\varepsilon}
$$

$L_{10}$ is the basic rated life span of the bearing, $C$ is the basic rated dynamic load $(\mathrm{N})$ of the bearing, $P$ is the equivalent dynamic load $(\mathrm{N})$, and $\varepsilon$ is the life index.

Considering the different reliability, unconventional

Table 2: Fatigue life test Data of Bearing

\begin{tabular}{lcccc}
\hline Bearing type & Group number & Number & $\beta$ Range & $\beta$ mean value \\
\hline Center ball bearing & 9 & 270 & $1.45 \sim 1.85$ & 1.69 \\
Deep groove ball bearing & 6 & 180 & $1.15 \sim 1.65$ & 1.42 \\
Cylindrical roller bearing & 3 & 90 & $0.95 \sim 1.35$ & 1.16 \\
Tapered roller bearing & 3 & 90 & $0.65 \sim 1.15$ & 0.88 \\
Spherical roller bearings & 4 & 120 & $0.45 \sim 1.05$ & 0.66 \\
\hline
\end{tabular}


material and operating conditions, the revised rated life span of the bearing is

$$
L(R)=\alpha_{1} \alpha_{2} \alpha_{3} L_{10}=\alpha_{1} \alpha_{2} \alpha_{3}\left(\frac{C}{P}\right)^{\varepsilon}
$$

$\alpha_{2}$ is the material coefficient, and the $\alpha_{3}$ is the lubrication coefficient, then

$$
C=\beta P[L(R)]^{\frac{1}{2}}
$$

The $\beta$ is the rating of the rated dynamic load reliability.

$$
\beta=\alpha^{-\frac{1}{\varepsilon}}=\left(\frac{\ln 0.9}{\ln R}\right)^{\frac{1}{\varepsilon k}}
$$

\subsection{Fuzzy interval valued reliability evaluation for colliery machine}

In the design and manufacture of mechanical parts, it not only contains randomness, but also includes fuzziness. The randomness and fuzziness can reflect the uncertainty of things, which is ambiguous, random uncertainty is due to the occurrence of event conditions cannot be controlled, resulting in some accidental factors that the test result is uncertain, or that is due to the causal laws of the insufficient, the connotation of both things is uncertain, while the extension is clear, and the fuzziness refers to is not clear phenomenon in reality, things that the extension is uncertain, but the meaning is clear.

The fuzziness existing in many engineering applications, but because of the limitation of conditions, because of the information itself and some factors is determined or random, it is not very clear, cannot be represented by determining the amount or amount of random, and this can be used to represent the fuzzy. When the problem of randomness is encountered in the product design, the problem of fuzziness will also be encountered. The classic reliability is the ability of the product to maintain the specified function in the prescribed time of use and under the prescribed use conditions. It is possible to realize the fuzzification of the function of the product by changing the maintenance function in the original definition into a certain function to some extent. The definition of fuzzy reliability is the extension of the definition of classical reliability. The definition of classical reliability is a special case of the definition of fuzzy reliability. Therefore, fuzzy reliability can be defined as the ability of a product to maintain certain functions in a certain extent within a prescribed time of use.

The interval valued fuzzy average method is to evaluate the reliability of colliery machine by replacing the average value of the traditional evaluation scheme with the effective interval score (Zhao, Guo and Yan, 2017).

The arithmetic of interval number can be shown by the following formula (Zhao, Cai and Fan, 2016).

$$
[R]=\left\{\left[a^{-}, a^{+}\right]: a^{-} \leq a^{+}, a^{-}, a^{+} \in R\right\}
$$

where $[R]$ is the set of all closed intervals formed on the real number set $\mathrm{R}$.

$$
\begin{aligned}
& \forall\left[a^{-}, a^{+}\right],\left[b^{-}, b^{+}\right] \in[R], a^{-}>0, b^{-}>0, \forall k \in Q, \\
& {\left[a^{-}, a^{+}\right]+\left[b^{-}, b^{+}\right]=\left[a^{-}+b^{-}, a^{+}+b^{+}\right]} \\
& k\left[a^{-}, a^{+}\right]=\left[k a^{-}, k a^{+}\right] \\
& \forall k_{i} \in Q,\left[a_{i}^{-}, a_{i}^{+}\right] \in[R], \text { then }
\end{aligned}
$$

$$
\left(k_{1}, k_{2}, \cdots k_{n}\right)\left(\begin{array}{l}
{\left[a_{1}^{-}, a_{1}^{+}\right]} \\
\vdots \\
{\left[a_{n}^{-}, a_{n}^{+}\right]}
\end{array}\right)=k_{1}\left[a_{1}^{-}, a_{1}^{+}\right]+\cdots k_{n}\left[a_{n}^{-}, a_{n}^{+}\right] .
$$

when $[a, b],[\mathrm{c}, \mathrm{d}] \in[R]$, then

$$
\begin{aligned}
& {[a, b]<[c, d], b<c} \\
& {[a, b] \prec[c, d], \frac{a+b}{2}<\frac{c+d}{2}, b \geq c} \\
& {[a, b]=[c, d], a=c, b=d} \\
& {[a, b] \approx[c, d], a \neq c, \frac{a+b}{2}=\frac{c+d}{2}} \\
& {\left[a^{-}, a^{+}\right]=\sum_{i=1}^{4} p_{i}\left[a_{i}^{-}, a_{i}^{+}\right]} \\
& F_{i j}=\left\{\begin{array}{l}
{\left[f_{i j 1}^{-}, f_{i j 1}^{+}\right]} \\
\vdots \\
{\left[f_{i j m}^{-}, f_{i j m}^{+}\right]}
\end{array}\right\}
\end{aligned}
$$

where $m$ is the number of three level indexes under the two level index $u_{i j}$. The weight can be calculated,

$R_{i j}=\left[r_{i j}^{-}, r_{i j}^{+}\right]=q_{i j} \times F_{i j}=\sum_{k=1}^{m} q_{i j k}\left[f_{i j k}^{-}, f_{i j k}^{+}\right]=\left[\sum_{k=1}^{m} q_{i j k} f_{i j k}^{-}, \sum_{k=1}^{m} q_{i j k} f_{i j k}^{+}\right]$

where $R_{i j}$ is the number of two level indexes under the first level index $u_{i j}$.

In the same way, the effective interval score of the first level index can be obtained

$$
R_{i}=\left[r_{i}^{-}, r_{i}^{+}\right]=\left[\sum_{j=1}^{m} q_{i j} r_{i j}^{-}, \sum_{j=1}^{m} q_{i j} r_{i j}^{+}\right]
$$

where, $m$ is the number of two level indexes under the first level index $u_{i}$

Effective interval score is

$$
R=\left[r^{-}, r^{+}\right]=\left[\sum_{i=1}^{m} q_{i} r_{i}^{-}, \sum_{i=1}^{m} q_{i} r_{i}^{+}\right]
$$

where $m$ is the number of primary indicators in the evaluation system.

The effective interval scores of the four evaluation subjects are as follows. 


\begin{tabular}{lllll}
\hline & \multicolumn{1}{c}{$\mathrm{G}_{1}$} & \multicolumn{1}{c}{$\mathrm{G}_{2}$} & \multicolumn{1}{c}{$\mathrm{G}_{3}$} & \multicolumn{1}{c}{$\mathrm{G}_{4}$} \\
\hline $\mathrm{A}_{1}$ & $<\left[\mathrm{s}_{2}, \mathrm{~s}_{3}\right],(0.5,0.6)>$ & $<\left[\mathrm{s}_{4}, \mathrm{~s}_{5}\right],(0.3,0.5)>$ & $<\left[\mathrm{s}_{2}, \mathrm{~s}_{3}\right],(0.7,0.8)>$ & $<\left[\mathrm{s}_{1}, \mathrm{~s}_{2}\right],(0.5)>$ \\
$\mathrm{A}_{2}$ & $<\left[\mathrm{s}_{3}, \mathrm{~s}_{4}\right],(0.3,0.4)>$ & $<\left[\mathrm{s}_{2}, \mathrm{~s}_{3}\right],(0.6)>$ & $<\left[\mathrm{s}_{4}, \mathrm{~s}_{5}\right],(0.3,0.4)>$ & $<\left[\mathrm{s}_{2}, \mathrm{~s}_{3}\right],(0.4)>$ \\
$\mathrm{A}_{3}$ & $<\left[\mathrm{s}_{2}, \mathrm{~s}_{3}\right],(0.4,0.5)>$ & $<\left[\mathrm{s}_{4}, \mathrm{~s}_{5}\right],(0.3,0.6)>$ & $<\left[\mathrm{s}_{2}, \mathrm{~s}_{3}\right],(0.1,0.5)>$ & $<\left[\mathrm{s}_{4}, \mathrm{~s}_{5}\right],(0.4,0.5)>$ \\
$\mathrm{A}_{4}$ & $<\left[\mathrm{s}_{5}, \mathrm{~s}_{6}\right],(0.2,0.3)>$ & $<\left[\mathrm{s}_{3}, \mathrm{~s}_{4}\right],(0.4)>$ & $<\left[\mathrm{s}_{1}, \mathrm{~s}_{2}\right],(0.4,0.7)>$ & $<\left[\mathrm{s}_{2}, \mathrm{~s}_{3}\right],(0.4,0.7)>$ \\
$\mathrm{A}_{5}$ & $<\left[\mathrm{s}_{2}, \mathrm{~s}_{3}\right],(0.4,0.5)>$ & $<\left[\mathrm{s}_{3}, \mathrm{~s}_{4}\right],(0.5,0.6)>$ & $<\left[\mathrm{s}_{1}, \mathrm{~s}_{2}\right],(0.4,0.6)>$ & $<\left[\mathrm{s}_{3}, \mathrm{~s}_{4}\right],(0.3,0.6)>$ \\
\hline
\end{tabular}

$\mathrm{Z}=\left[\mathrm{z}^{-}, \mathrm{z}^{+}\right]=\left[\sum_{\mathrm{i}=1}^{4} \mathrm{~W}_{\mathrm{i}} \mathrm{z}_{\mathrm{i}}^{-}, \sum_{\mathrm{i}=1}^{4} \mathrm{~W}_{\mathrm{i}} \mathrm{z}_{\mathrm{i}}^{+}\right]$

Step 1. Suppose the fuzzy measure of attribute $G_{j}(j=1,2, \ldots, n)$ and attribute sets of as follows:

$\mu\left(G_{1}\right)=0.30, \mu\left(G_{2}\right)=0.24, \mu\left(G_{3}\right)=0.29, \mu\left(G_{4}\right)=0.20$

$\mu\left(G_{1}, G_{2}\right)=0.62, \mu\left(G_{1}, G_{3}\right)=0.53, \mu\left(G_{1}, G_{4}\right)=0.50, \mu\left(G_{2}, G_{3}\right)=0.40$

$\mu\left(G_{2}, G_{4}\right)=0.45, \mu\left(G_{3}, G_{4}\right)=0.50, \mu\left(G_{1}, G_{2}, G_{3}\right)=0.70, \mu\left(G_{1}, G_{2}, G_{4}\right)=0.80$

$\mu\left(G_{1}, G_{3}, G_{4}\right)=0.74, \mu\left(G_{2}, G_{3}, G_{4}\right)=0.68, \mu\left(G_{1}, G_{2}, G_{3}, G_{4}\right)=1.00$

Step 2. The complex character involving the opinion of different decision-makers.

Step 3. We used the decision information given in matrix $H$ and the IHFULCA operator to obtain the scores

\begin{tabular}{lllll}
\multicolumn{5}{c}{ TABLE 4: INDUCING VARIables } \\
\hline & $\mathrm{G}_{1}$ & $\mathrm{G}_{2}$ & $\mathrm{G}_{3}$ & $\mathrm{G}_{4}$ \\
\hline $\mathrm{A}_{1}$ & 16 & 14 & 18 & 11 \\
$\mathrm{~A}_{2}$ & 20 & 22 & 21 & 16 \\
$\mathrm{~A}_{3}$ & 16 & 15 & 20 & 17 \\
$\mathrm{~A}_{4}$ & 13 & 19 & 13 & 16 \\
$\mathrm{~A}_{5}$ & 18 & 20 & 17 & 22 \\
\hline
\end{tabular}

$S\left(\tilde{h}_{i}\right)(i=1,2,3,4,5)$ of the overall fuzzy uncertain preference values $h_{i}(1,2,3,4,5)$.

$$
\begin{aligned}
& S\left(\tilde{h}_{1}\right)=\left[\mathrm{s}_{1.21}, \mathrm{~s}_{2.14}\right], S\left(\tilde{h}_{2}\right)=\left[\mathrm{s}_{2.56}, \mathrm{~s}_{3.78}\right], S\left(\tilde{h}_{3}\right)=\left[\mathrm{s}_{0.74}, \mathrm{~s}_{1.35}\right] \\
& S\left(\tilde{h}_{4}\right)=\left[\mathrm{s}_{1.28}, \mathrm{~s}_{3.78}\right], S\left(\tilde{h}_{5}\right)=\left[\mathrm{s}_{0.75}, \mathrm{~s}_{3.59}\right]
\end{aligned}
$$
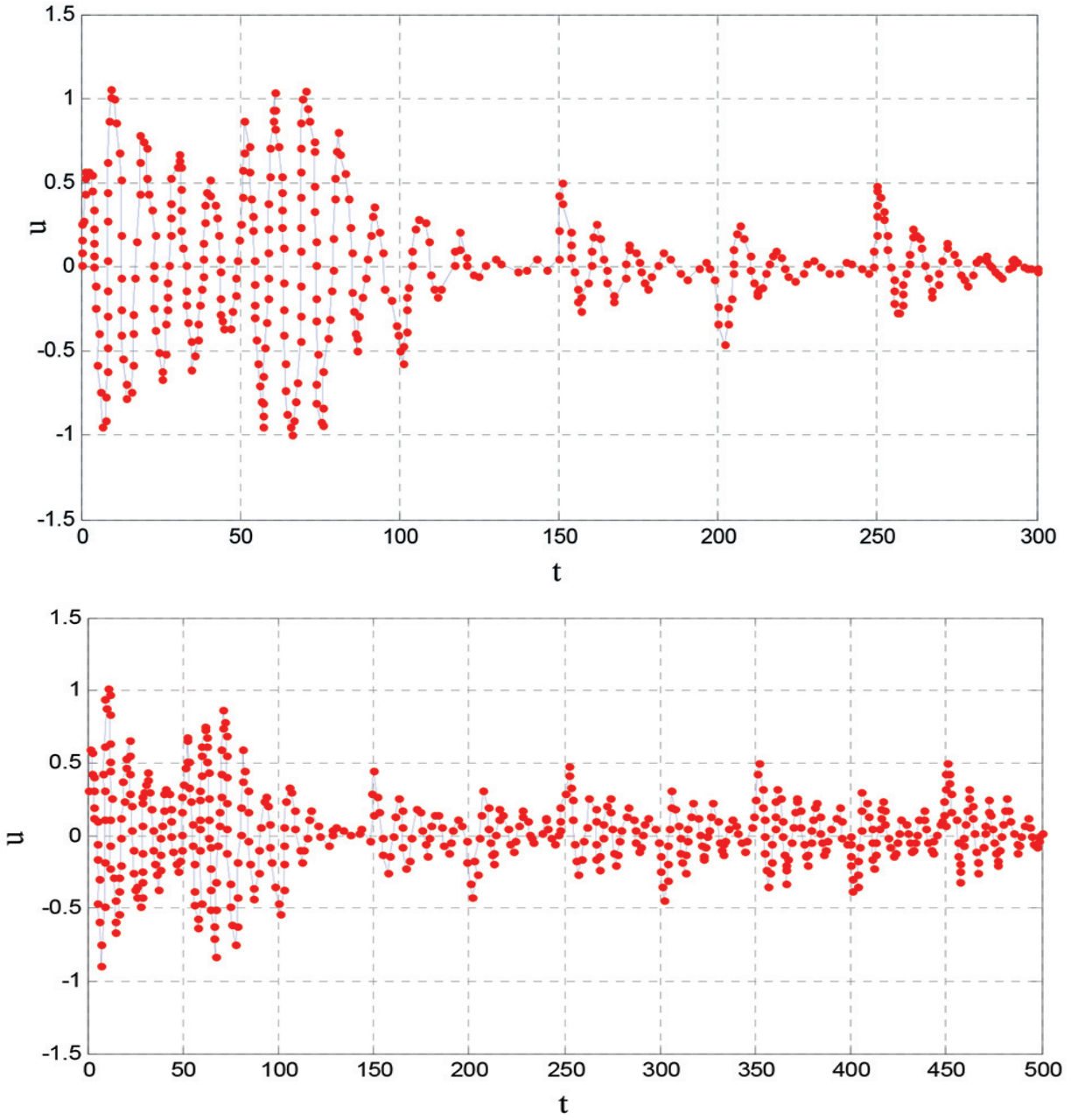

Step 4. The enterprise innovation fluctuationis in accordance with the scores.

The results are shown in Table 4.

The mechanical reliability curve is shown as Figs. 1 and 2.

Fig.1 Mechanical reliability curve without fuzzy interval evaluation

Fig.2 Mechanical reliability curve with fuzzy interval evaluation 


\subsection{Conclusion}

The accuracy and advanced nature of the study of reliability design is based on the authenticity and accuracy of the data of stress, strength and life. The main difficulty is the lack of effective research on the reliability of the data, therefore, pay attention to the collection and analysis of the test data, can accurately reflect the physical characteristics of the product design data, design of new products have great reference value, is another direction of research on reliability design of mechanical products.

\section{Acknowledgement}

This work is financially supported by the National Natural Science Foundation Guidance Project of Liaoning Province (NO. 201602650), and the Scientific Research Project of Department of Education of Liaoning Province (NO.L2015469.)

\section{References}

1. P Karthikeyan, S Vasuki. (2016): Hybrid Approach of Efficient Decision-Based Algorithm and Fuzzy Logic for the Removal of High Density Salt and Pepper Noise in Images. Journal of Circuits Systems \& Computers, 25 (10), 130-145.

2. MA Butt, M Akram. (2016): A Novel Fuzzy DecisionMaking System for CPU Scheduling Algorithm. Neural Computing \& Applications, 27 (7), 1-13.

3. A Pahlavani. (2017): A Hybrid Algorithm Of Improved Case-Based Reasoning And Multi-Attribute Decision Making In Fuzzy Environment For Investment Loan Evaluation. International Journal of Information \&
Decision Sciences, 2 (1), 17-49.

4. Y Zhao, Y Cai, Q Song (2018): Energy control of plugin hybrid electric vehicles using model predictive control with route preview. IEEE/CAA Journal of Automatica Sinica, DOI: 10.1109/JAS.2017.7510889, 1-8.

5. Yang Zhao, Zhenghong Guo, Jianming Yan. (2017): Vibration Signal Analysis and Fault Diagnosis of Bogies of the High-speed Train based on Deep Neural Networks. Journal of Vibroengineering, 19(4), 2456-2474.

6. MAB Messaoud, A Bouzid, N Ellouze. (2016): A New Biologically Inspired Fuzzy Expert System-Based Voiced/Unvoiced Decision Algorithm for Speech Enhancement. Cognitive Computation, 8 (3), 478-493.

7. Chen Shyi-Ming, Lee Chia-Hoang. (2014): New Methods for Students' Evaluation using Fuzzy Sets. Fuzzy Sets and Systems, 10(2), 200-218.

8. Ghondaghsaz, Fatemeh, Rasekh, Abdolrahman. (2012): Case-Deletion Diagnostics for Testing a Linear Hypothesis about Weighted Regression Coefficients. International Journal of Intelligent Technologies \& Applied Statistics, 5(3), 237-248.

9. F Tiryaki, B Ahlatcioglu. (2015): Fuzzy Portfolio Selection using Fuzzy Analytic Hierarchy Process. Information Sciences, 179 (2), 53-69.

10. Yang Zhao, Yanguang Cai, Guobing Fan. (2016): Dynamical Behaviour for Fractional-order Shunting Inhibitory Cellular Neural Networks. Journal of Nonlinear Science and Applications, 9(6), 4589-4599.

\section{Subscribe to :}

INDIAN JOURNAL OF POWER \& RIVER VALLEY DEVELOPMENT

A technical monthly devoted to development of POWER AND WATER RESOURCES IN ASIA

Annual Subscription : Rs.3000 (India); $£ 280.00$ or $\$ 400.00$ (Foreign)

For copies please contact:

The Manager

Books \& Journals Private Limited

Moon Plaza (2A, 2nd Floor), 62 Lenin Sarani, Taltala, Kolkata 700013

e-mail: bnjournals@gmail.com; pradipchanda@yahoo.co.uk;

www.jmmf.info •Mob: +919239384829 